\author{
Alfonso Arellano Espinar* \\ Luis Díez Catalán** \\ Roberto Alejandro Neut Smith ${ }^{* *}$
}

\title{
REVOLUCIÓN TECNOLÓGICA Y DESARROLLO SOCIOECONÓMICO: UNA AGENDA PARA EL MERCADO DE TRABAJO
}

El avance digital que estamos viviendo en la actualidad impacta e impactará tanto a los trabajadores como a las empresas de forma muy heterogénea. En este artículo mostramos evidencia comparada en el mercado laboral de España y planteamos una propuesta que permita a todos los agentes beneficiarse de este avance digital, orientada en tres directrices: formación, adaptación de las instituciones laborales y reducción de las posibles desigualdades derivadas de este impacto heterogéneo.

\section{Technological revolution and socioeconomic development: an agenda for the labour market}

The digital advances we are currently experiencing are impacting and will continue to impact both workers and businesses in a highly heterogeneous manner. In this article, we present comparative evidence in the labour market in Spain and put forward a proposal that will allow all actors to benefit from these digital advances, focused around three axes: education, the adaptation of labour institutions and the reduction of the potential inequalities arising from this heterogeneous impact.

Palabras clave: disrupción tecnológica, vacantes, formación, desigualdad.

Keywords: technological disruption, vacancies, education, inequality.

JEL: J24, J63, O33.

1. Introducción

Dos tecnologías lideran el avance digital y prometen continuar reconfigurando el mapa ocupacional en España, Europa y el mundo. La primera es la plataforma digital, un punto de encuentro virtual donde almacenar

\footnotetext{
* BBVA Research y Universidad Complutense de Madrid.

** BBVA Research.

Versión de octubre de 2019.

DOI: https://doi.org/10.32796/ice.2019.911.6942
}

datos e intercambiar todo tipo de información, bienes y servicios, y la segunda es la inteligencia artificial, una tecnología predictiva que bebe de la ingente cantidad de datos que genera la digitalización de la información. Si bien ambas tecnologías no son nuevas, especialmente en el caso de la inteligencia artificial cuyos inicios datan de mediados del siglo $X X$ a partir del trabajo seminal de Turing (1950), solo en los últimos años ambas han proliferado a través de una multiplicidad de aplicaciones por parte de empresas, gobiernos y personas. Se observa la ubicuidad de diversas plataformas en las redes sociales, 
la economía y financiación colaborativa, y la creciente omnipresencia de la inteligencia artificial detrás del reconocimiento automático de imágenes, idiomas, dolencias y perfiles personales. La difusión y aplicación de estas tecnologías solo irá a más, consolidándose un nuevo estadio de la «revolución digital» que empezó a finales del siglo XX con la aparición de internet.

Como en revoluciones tecnológicas pasadas, el impacto sobre el mercado laboral será profundo y generará importantes modificaciones en el contenido del trabajo y la forma de trabajar. En cuanto al contenido, ligado tanto a las ocupaciones que se automatizarán como a las que se crearán, el impacto dependerá por un lado de la tecnología que disponga la industria y por otro de las capacidades que adquiera la población activa. Respecto a la forma, que incluye el tipo de relación laboral (temporal o indefinida, a tiempo completo o a tiempo parcial), la organización entre trabajadores, la distribución de retornos generados, requisitos de seguridad y prestaciones sociales, será producto no solo de la tecnología implementada y las capacidades adquiridas, sino también de los modelos de negocio creados, la negociación entre agentes económicos y el marco institucional.

Empezando por el contenido, ¿en qué ocupaciones se trabajará? Predecir las actividades que se crearán en las próximas décadas es un ejercicio de futurología con altísima incertidumbre y en el que se suele subestimar el ingenio humano. ¿Cómo haber predicho a los diseñadores de aplicaciones a principios del siglo XXI, cuando los móviles contaban inicialmente con un teclado y una pantalla solo para números? Y a más largo plazo, ¿cómo alguien en el mundo eminentemente rural y no electrificado de 1900 pudo haber predicho las ocupaciones en las industrias del cine y la televisión, el transporte y el turismo que prevalecerían 50 años más tarde? Como consecuencia de esta incertidumbre, siempre ha surgido el temor a un mundo totalmente automatizado donde el trabajador sea obsoleto. Pero esto es algo que hasta la fecha no se ha producido, y que basándose en estudios recientes como el del premio nobel de Economía William Nordhaus (2015), podemos continuar descartando para este siglo.
Sin embargo, este optimismo no debe transformarse en complacencia. La potencial creación de nuevas ocupaciones es la contraparte a la clara obsolescencia de otras. Doménech et al. (2018) estiman que, dada las nuevas tecnologías, hasta un $37 \%$ de las actuales ocupaciones en España corren el riesgo de ser automatizadas. Es un porcentaje inferior al $47 \%$ de ocupaciones en riesgo estimado para EE UU (Frey y Osborne, 2017), y menor a la proporción de ocupaciones en riesgo en países emergentes (en torno al 40 y 50 \%, según Belapatiño et al., 2019), pero especialmente significativo para un país que ostenta una tasa de desempleo superior al $15 \%$ en 2018. Además, aunque las revoluciones tecnológicas del pasado dan soporte al optimismo de cara al largo plazo, estas evidenciaron fuertes disrupciones del mercado laboral en el corto y medio plazo. Mokyr et al. (2015) resaltan el perjuicio de la primera revolución industrial para toda una generación de trabajadores en sectores claves de la economía (e. g., industria textil), pero no tanto en términos de desempleo como en términos de menores sueldos, mayor precariedad (las revueltas luditas fueron más un reclamo por mejores condiciones de trabajo que por la preservación de puestos de trabajo) y un creciente temor a la alienación laboral.

La mala gestión de esta transición puede conllevar graves secuelas, pues los colectivos que ven sus ocupaciones desaparecer, no son los mismos a los que se les abren nuevas oportunidades. Esto es porque más que cambios en las ocupaciones existentes, lo que la tecnología trae son cambios en las habilidades y capacidades necesarias para desarrollar distintos oficios. La disrupción digital presentaría pocos problemas si ocupaciones nuevas y obsoletas requiriesen las mismas habilidades, ya que el único desafío sería asegurar una intermediación eficaz para emparejar trabajadores con las nuevas oportunidades laborales. Pero no es así, y por ello una buena gestión de la transición pasa necesariamente por facilitar una buena formación y recapacitación en aquellas habilidades y capacidades necesarias para la era digital. 


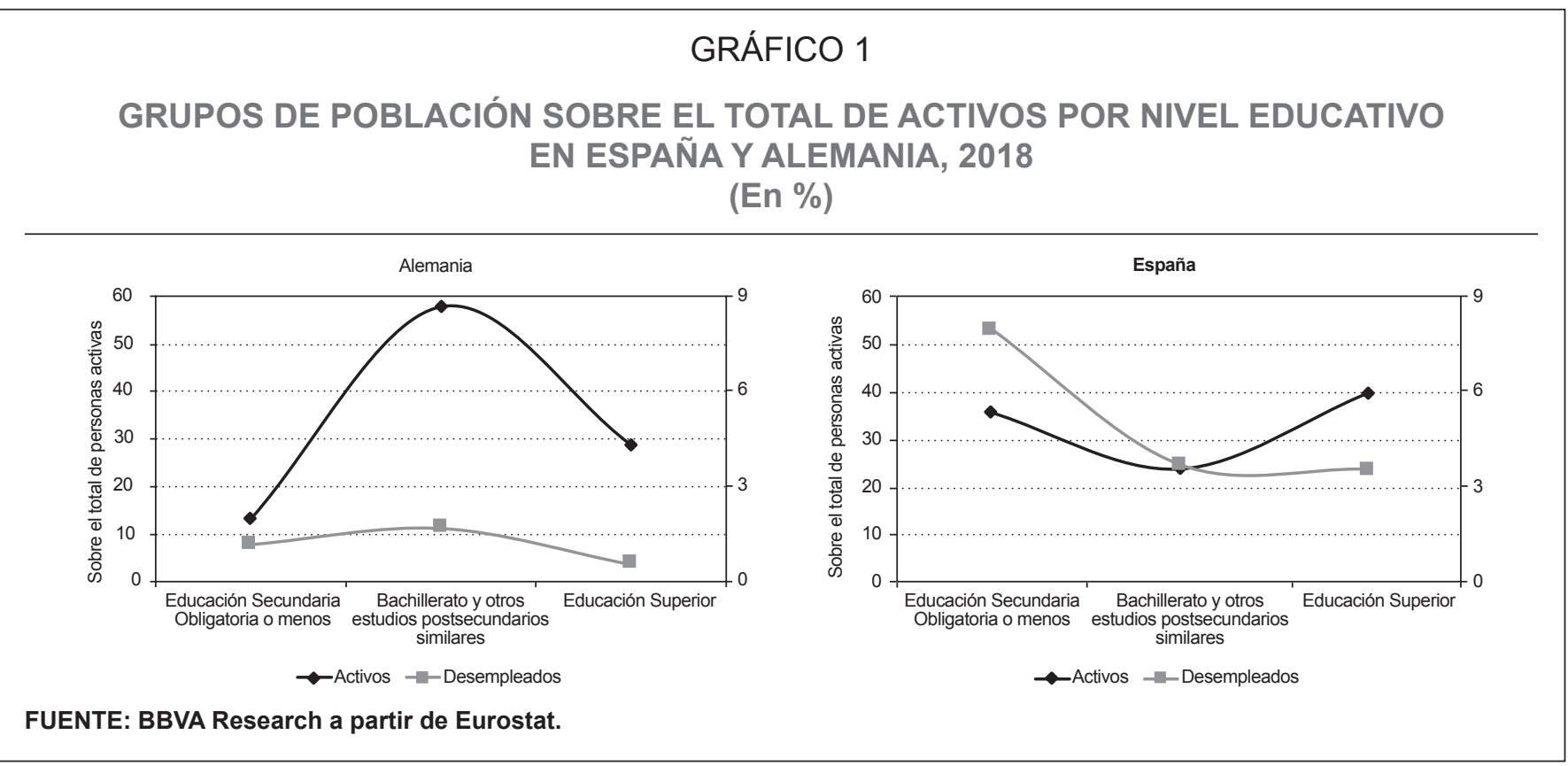

La importancia de esta transición está condicionada además por la necesidad de estimular un crecimiento inclusivo que nos permita reducir la desigualdad. Según la evidencia que muestran Andrés et al. (2019) al relacionar el nivel de desigualdad de Gini con la renta bruta nacional para un grupo de países de la OCDE, España no parece encontrarse en la frontera de posibilidades y tener por ello que escoger entre mayor crecimiento o menor desigualdad. A diferencia de otros países europeos con un índice de Gini del 30 \% o menos, como son los nórdicos y Alemania, España muestra un índice de desigualdad relativamente elevado (alrededor de 35 sobre cien), muy similar a Portugal y Grecia. Por otro lado, la renta bruta nacional per cápita en España es un 60 \% del referente de EE UU, similar a la de Italia y por debajo de Alemania y los países del norte de Europa, entre el 80 y el $90 \%$. Esta discrepancia justifica un estudio comparado con mayor detalle de estos dos perfiles - España con menor renta y mayor desigualdad que varios países europeos, y Alemania con altos niveles de renta y bajos niveles de desigualdad.

El resto del artículo se distribuye de la siguiente manera: en el segundo apartado nos centramos en una comparación más detallada entre el talento y la formación en España y lo que se observa para el mercado laboral alemán; en el tercer apartado estudiamos en qué sectores hay demanda no satisfecha de talento y qué tipo de talento y habilidades escasea; posteriormente, en el cuarto apartado proponemos estrategias de política para superar este reto; y finalmente, el quinto apartado presenta las principales conclusiones del artículo.

\section{Papel de la formación en el mercado laboral de España}

¿De qué habilidades y capacidades dispone hoy la fuerza laboral en España? Analizando aquellas capacidades adquiridas a través de la formación reglada, se observan dos características de especial relieve cuando se compara España al paradigma alemán, un país europeo que se encuentra en la frontera tecnológica, con bajo desempleo, alta productividad y un mercado laboral agregado sorprendentemente resiliente durante la última gran crisis global. Primero, la fuerza laboral en España cuenta con una fuerte dualidad formativa. Como muestra el Gráfico 1, la distribución de 


\begin{tabular}{|c|c|c|c|c|}
\hline & DE D & EO POR NIVEL E & $\begin{array}{l}\text { RO } 1 \\
\text { ATIVO EN ESPAÑA Y } \\
\text { Y) }\end{array}$ & MANIA, 2018 \\
\hline & Total & $\begin{array}{l}\text { Educación Secundaria } \\
\text { Obligatoria o menos }\end{array}$ & $\begin{array}{l}\text { Bachillerato y otros estudios } \\
\text { postsecundarios similares }\end{array}$ & Educación Superior \\
\hline Alemania & 3,5 & 9,0 & 2,9 & 1,9 \\
\hline España & 15,4 & 22,3 & 15,5 & 9,0 \\
\hline
\end{tabular}

la población activa en España tiene forma de parábola, concentrada en los extremos de baja y alta formación. Por el contrario, en Alemania se aprecia una parábola invertida, en la que la mayor parte de la población activa presenta un nivel de educación medio. Segundo, el elevado desempleo en España es mucho más acuciante para la fuerza laboral poco formada, pero la brecha salarial con respecto a Alemania perjudica mucho más a la fuerza laboral española de alta formación.

En relación con el primer punto, para ambos países, los brazos de las parábolas son asimétricos en longitud y muestran que la fuerza laboral de más elevada formación tiene un tamaño mayor que la fuerza laboral de más baja formación, una realidad muy diferente a la de países en desarrollo. Es más, en España, un $40 \%$ de la fuerza laboral es altamente cualificada (estudios universitarios o superior), mientras que esta cifra llega a solo al $29 \%$ en Alemania. Sin embargo, en línea con la dualidad española, el $36 \%$ de la fuerza laboral española solo cuenta con el mínimo de formación requerida por ley, una cifra que en Alemania es de apenas un $13 \%$. En definitiva, España debe avanzar en la formación de la fuerza laboral, pero los retornos no están en fomentar una mayor formación universitaria, sino en facilitar una formación intermedia a aquellos que solo alcanzan el mínimo legal.

En relación con el segundo punto, al observar las diferencias en el desempleo por nivel educativo (Cuadro 1), en ambos países la tasa de desempleo es más elevada para la fuerza laboral menos formada. Un resultado que no sorprende, dado que un trabajador con mayor formación puede sortear la situación de desempleo aceptando un puesto con menos requerimientos formativos de los que tiene en su haber. En este caso, el desempleo se esconde tras la sobrecualificación. Alemania, con una tasa de desempleo general del $3,5 \%$ en 2018 , muestra destacables diferencias entre la tasa de desempleo de la población menos formada ( $9 \%$ ) y el resto de niveles educativos $(2,9 \%$ para aquellos con un nivel medio y $1,9 \%$ para los que cuentan con estudios universitarios o superiores). En el caso de España, esta tendencia se repite, pero de manera mucho más acentuada: con una tasa de desempleo general del $15,4 \%$ en 2018 , la fuerza laboral menos formada presenta una tasa de desempleo del $22,3 \%$ frente a una tasa del $15,5 \%$ para aquellos con un nivel medio y del $9 \%$ para los que cuentan con título universitario o superior. Aunque estos niveles dependen del ciclo económico, las brechas existentes entre las tasas de desempleo de los distintos niveles educativos parecen ser estructurales, ya que prácticamente no han cambiado en el ciclo desde 2009: alrededor de 8 puntos porcentuales separan las tasas de desempleo del colectivo con menor nivel educativo de las de la gente de nivel medio, y otros 7 puntos porcentuales separan el desempleo entre el nivel medio y el superior. 


\section{CUADRO 2}

\begin{tabular}{|c|c|c|c|c|c|}
\hline & Total & $\begin{array}{c}\text { Educación } \\
\text { Secundaria } \\
\text { Obligatoria o } \\
\text { menos }\end{array}$ & $\begin{array}{l}\text { Bachillerato y } \\
\text { otros estudios } \\
\text { similares }\end{array}$ & $\begin{array}{l}\text { Educación } \\
\text { Superior }\end{array}$ & $\begin{array}{l}\text { Máster y } \\
\text { Doctorado }\end{array}$ \\
\hline $\begin{array}{l}\text { Industria, construcción y servicios ......................... } \\
\text { (excepto act. del hogar y act. de instituciones } \\
\text { y organismos extraterritoriales) }\end{array}$ & 64,39 & 83,74 & 65,73 & 61,34 & 57,81 \\
\hline $\begin{array}{l}\text { Industria, construcción y servicios ...................... } \\
\text { (excepto AA PP y defensa; Seguridad Social } \\
\text { obligatoria) }\end{array}$ & 64,40 & 83,47 & 64,82 & 60,23 & 56,23 \\
\hline Industria & 59,67 & 78,63 & 66,43 & 55,10 & 50,11 \\
\hline 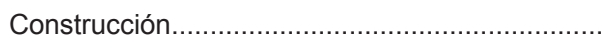 & 69,50 & 95,65 & 66,26 & 62,28 & 64,12 \\
\hline $\begin{array}{l}\text { Servicios } \\
\text { (excepto act. del hogar y act. de instituciones y } \\
\text { organismos extraterritoriales) }\end{array}$ & 66,20 & 84,53 & 67,32 & 66,84 & 59,72 \\
\hline Servicios .... & 66,48 & 84,13 & 66,18 & 65,40 & 58,26 \\
\hline
\end{tabular}

(excepto AA PP y defensa; Seguridad Social

obligatoria)

NOTA: Un porcentaje del $100 \%$ implica que los ingresos mensuales medios de España coinciden con los de Alemania para el grupo considerado. Los ingresos mensuales medios cubren la remuneración en efectivo pagada antes de cualquier deducción fiscal y de las cotizaciones a la Seguridad Social a cargo de los asalariados y retenidas por el empleador, y se limitan a los ingresos brutos que se pagan en cada período de pago durante el mes de referencia.

FUENTE: BBVA Research a partir de Eurostat (Encuesta de Estructura Salarial Europea).

En definitiva, nos encontramos ante dos países, cada uno con una estructura productiva y poblacional diferente en términos educativos, en los que las tasas de desempleo, aunque a diferentes niveles, son más acuciantes en la población menos formada.

Sería incorrecto detenerse en este resultado y concluir que el desajuste laboral se concentra en la parte de la población con menor formación. Como se ha observado inicialmente, un desajuste entre oferta y demanda en el mercado laboral puede reflejarse por vías distintas al desempleo, por ejemplo, a través de la sobrecualificación. Al no disponer de datos directos, nos acercamos al análisis a través de las encuestas de estructura salarial.

Como se aprecia en el Cuadro 2, el ingreso mensual medio en España es dos tercios del que hay en
Alemania, una brecha relativamente estable para los distintos sectores productivos de la economía, pero que presenta diferencias importantes según el nivel de formación. Las personas con menor nivel de educación en España tienen ingresos mensuales medios relativamente cercanos a los de trabajadores con similar formación en Alemania (equivalente al $85 \%$ ). Sin embargo, esta brecha crece significativamente a medida que aumenta el nivel de educación reglada, situándose por debajo del $60 \%$ al comparar a trabajadores con máster o doctorado. Este resultado es congruente con la existencia de un fenómeno de sobrecualificación o subempleo en España, donde los trabajadores con más nivel de educación tienen una menor tasa de desempleo, pero la ausencia de 
puestos de trabajo adecuados a su formación les obliga a ocupar una posición inferior, con las correspondientes consecuencias en términos salariales. Por ejemplo, Bell y Blanchflower (2018) estiman una tasa de subempleo en España del 22,7 \% en 2016, muy por encima de las tasas de Alemania $(5,8 \%)$, Francia $(15,7 \%)$ o Italia (13\%).

En definitiva, el desajuste entre oferta y demanda laboral en España no es un problema que atañe solo a trabajadores con poca formación. Aunque se observen mayores tasas de desempleo entre aquellos menos formados, un problema en parte exacerbado por la cota salarial que impone el salario mínimo legal, tanto el déficit de productividad como el desajuste laboral es palpable en todo el espectro formativo de la población activa. Este es un fenómeno que se puede acentuar con una mala gestión de la transformación tecnológica.

Dada la dualidad formativa en la población activa española, y los desajustes ya existentes en ambos extremos del espectro formativo, una buena gobernanza de la digitalización debe velar por una transición eficaz en todos los niveles formativos. En un extremo, deben realizarse esfuerzos adicionales en formación de nivel medio que permitan reducir la elevada fracción de población activa que cuenta con baja formación y que se encuentra fuertemente desempleada. En el otro extremo, es necesario disminuir el desajuste laboral para niveles educativos elevados que muestran un fuerte déficit de productividad. Un déficit que, como se verá a continuación, no es solo producto de un mal aprovechamiento del talento, sino también producto de un sistema educativo que no está alineado con las capacidades y habilidades requeridas por la economía del siglo XXI.

\section{Claves para entender las necesidades de talento en la economía española}

Con el objetivo de poder esclarecer el grado de desacoplamiento entre oferta y demanda de trabajo en España comparamos las tasas de vacantes entre sus distintos sectores de actividad. La tasa de vacantes se define como la ratio de plazas vacantes de empleo sobre el total de plazas ofertadas en un sector (estén estas ocupadas o vacantes). El Gráfico 2 muestra una correlación positiva entre esta tasa y el nivel de educación medio para los distintos sectores productivos que conforman la economía española.

Este resultado pone de relieve la existencia de una demanda por talento no cubierta en la economía, un problema más acusado en aquellos sectores más intensivos en capital humano altamente cualificado. Además, como se muestra en el Gráfico 3, dicha demanda no satisfecha también tiende a ser superior en aquellos sectores que han liderado el crecimiento económico de las últimas dos décadas (medido por el crecimiento en el peso de estos sectores en el PIB de la economía). Ambas correlaciones apuntan en una misma dirección, apoyan la tesis de escasez de talento altamente cualificado $\mathrm{y}$, además, sugirieren que dicha escasez es más pronunciada en los sectores que han liderado el crecimiento de las dos últimas décadas. Más que un problema de mala utilización de dicho talento, esta evidencia de vacantes nos muestra que la economía digital (sobrerrepresentada en los sectores que más crecen) tiene problemas para encontrar capacidades en la fuerza laboral altamente cualificada de España.

La piedra de toque que puede permitir realizar una transición adecuada entre las formas de trabajo establecidas y creadas en el siglo XX, y las nuevas oportunidades y los retos que conlleva el siglo XXI, se centra en identificar y proporcionar las habilidades y el conocimiento necesario para crecer en la economía digital. Existe abundante evidencia de un empuje por conocimientos en Ciencias, Tecnologías, Ingeniería y Matemáticas (STEM, por sus siglas en inglés). Díez et al. (2018) muestran qué ocupaciones intensivas en el uso de habilidades informáticas de hardware y software conllevan mayores salarios. Sin embargo, las habilidades requeridas por el sistema productivo siempre han ido más allá del mero dominio de la lógica, 


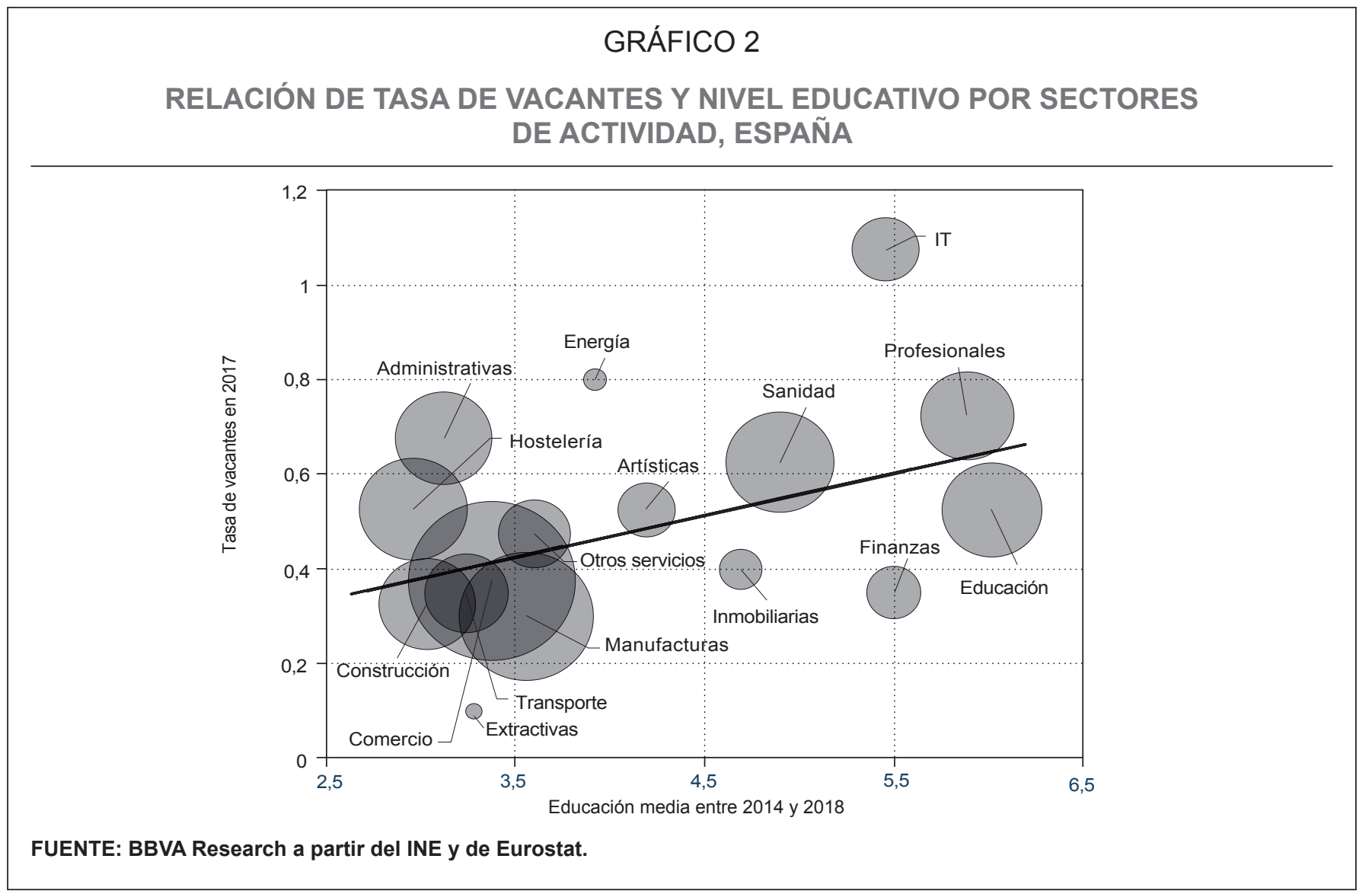

las matemáticas o la lingüística. Existen habilidades asociadas a inteligencias menos analíticas, como las habilidades emocionales asociadas a la inteligencia intrapersonal e interpersonal, y habilidades artísticas propias de la inteligencia creativa. Estas habilidades van siendo cada vez más demandadas por empresas en diferentes sectores económicos.

El análisis anterior muestra que los sectores que lideran el crecimiento tienen mayor dificultad para cubrir sus plazas pero, ¿en la búsqueda de qué talento específico tienen problemas? ¿Qué habilidades escasean y cuáles abundan en España? El Cuadro 3 resume los resultados al correlacionar la intensidad de capital humano altamente cualificado con la «escasez» de una batería de capacidades específicas. Para la medición de escasez se utiliza un índice de escasez / sobreabundancia de distintos talentos elaborado por la OCDE para los diferentes sectores de actividad. Los talentos identificados incluyen varios tipos de conocimientos, capacidades, competencias, habilidades y formas de colaboración y trabajo. Ejemplos de talentos que escasean en muchos sectores de la economía española van desde la destreza en "habilidades cuantitativas» hasta la «adaptabilidad» en el trabajo o la capacidad de realizar «trabajo independiente». El Cuadro 3 pone en evidencia que, para cada uno de los talentos identificados, la escasez aumenta significativamente en aquellos sectores más intensivos en el uso de capital humano altamente cualificado - sectores que sostienen los trabajos mejor remunerados y que además han liderado el crecimiento en estas primeras décadas de la revolución digital. Esta mayor escasez en sectores líderes es especialmente pronunciada cuando nos centramos en los talentos considerados "sospechosos habituales», 


\section{GRÁFICO 3}

\section{RELACIÓN DE TASA DE VACANTES Y CRECIMIENTO DEL VALOR AÑADIDO BRUTO (VAB) POR SECTORES DE ACTIVIDAD, ESPAÑA}

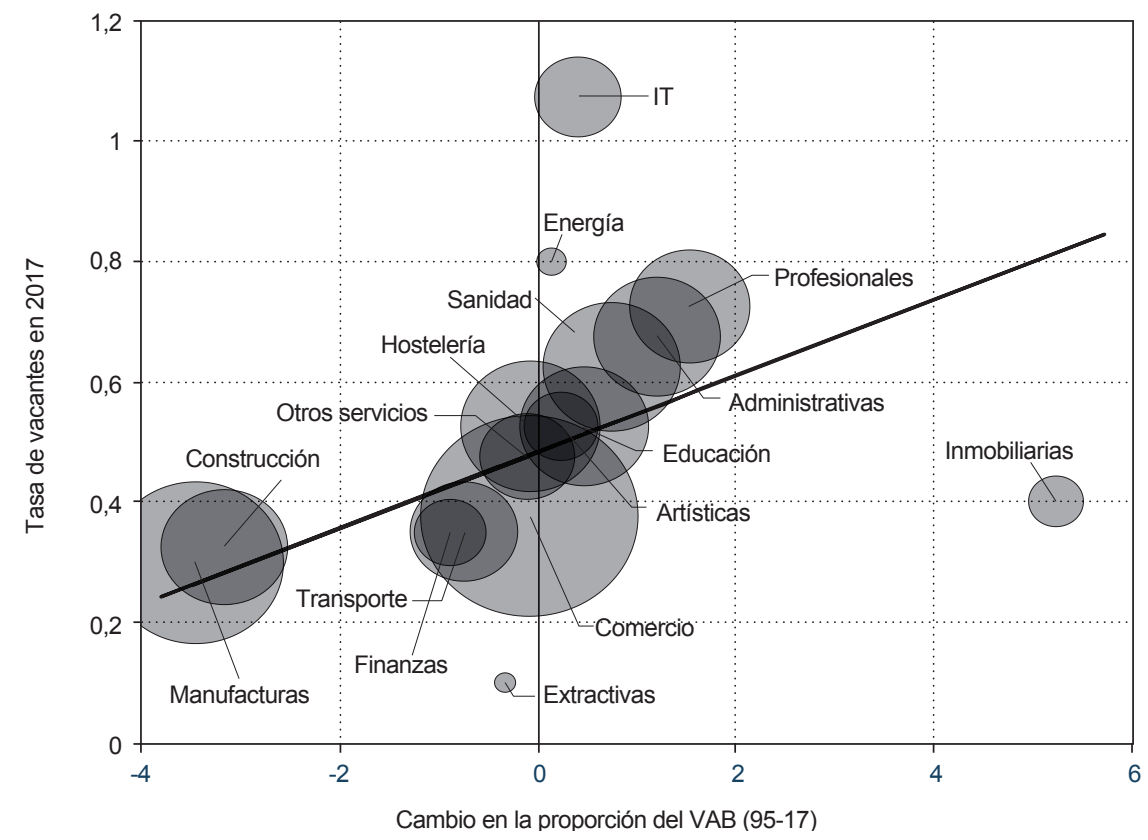

FUENTE: BBVA Research a partir del INE y de Eurostat.

como la «capacidad de resolver problemas complejos» y «conocimientos de matemáticas y ciencia», pero también sucede con la mayoría de las competencias de carácter interpersonal y social como «competencias sociales», la «inteligencia práctica» y la capacidad de realizar «trabajo independiente».

En otras palabras, se detecta un claro desajuste entre la demanda y la oferta de capacidades que se antoja más acusado en los sectores protagonistas del crecimiento del siglo XXI, un problema que no se limita a la cantidad de formación reglada tradicional ya que la escasez incluye capacidades que no se desarrollan bajo el sistema educativo actual. Esto refuerza la idea de que las transformaciones asociadas al cambio tecnológico están empezando a impactar en la economía española y que deberíamos preocuparnos no solo por la mejora de los contenidos tradicionales en la formación reglada, sino que también tendríamos que potenciar el aprendizaje continuo de otras actividades y capacidades que la nueva economía de servicios demanda.

La escasez relativa de ciertas habilidades no es solo un escollo para la productividad general, también puede agravar la desigualdad entre trabajadores. Tomemos como ejemplo los conocimientos y habilidades asociadas al mundo de la informática. Como hemos explicado anteriormente, Díez Catalán et al. (2018) muestran que el salario promedio de una ocupación aumenta con el grado de informatización ${ }^{1}$ de dicha ocupación. Igualmente, estas ocupaciones también tienden a tener un menor nivel de rutinización y, por tanto, un menor riesgo de automatización. En este contexto, es clave la

\footnotetext{
1 Medido como el número de habilidades informáticas necesarias para
} desempeñar dicha ocupación. 


\section{CUADRO 3}

\section{ESTIMACIONES EN LA RELACIÓN ENTRE NECESIDAD DE ACTITUDES Y APTITUDES Y SECTORES ECONÓMICOS, ESPAÑA, 2015}

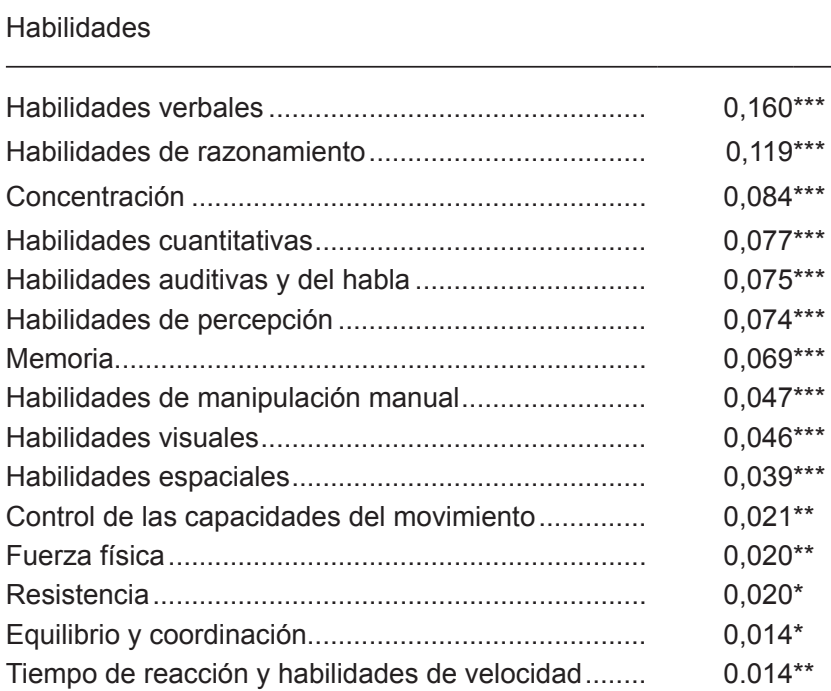

Conocimientos

\begin{tabular}{|c|c|}
\hline 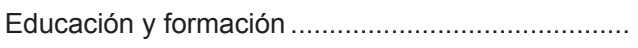 & $0,142^{* * *}$ \\
\hline Negocios y administración...................................... & $0,095^{* * *}$ \\
\hline Derecho y seguridad pública ...................................... & $0,068^{* * *}$ \\
\hline Matemáticas y ciencia .................... & $0,058^{* * *}$ \\
\hline Ingeniería y tecnología .............. & $0,053^{* * *}$ \\
\hline Comunicación................................ & $0,050^{* * *}$ \\
\hline Manufacturas y producción ........... & $0,037^{* * *}$ \\
\hline 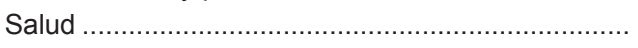 & $0,035^{\star *}$ \\
\hline Arte y humanidades.... & $0,032^{*}$ \\
\hline
\end{tabular}

Formas de colaboración y trabajo

\begin{tabular}{|c|c|}
\hline Adaptabilidad ............... & $0,283^{* * *}$ \\
\hline Independencia ....................... & $0,272^{* * *}$ \\
\hline Orientación al logro ...... & $0,266^{* * *}$ \\
\hline Influencia social ................ & $0,253^{* * *}$ \\
\hline Concienciación .......................... & $0,252^{* * *}$ \\
\hline 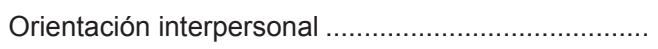 & $0,246^{* * *}$ \\
\hline Inteligencia práctica.......... & $0,239 * * *$ \\
\hline
\end{tabular}

NOTA: En el Cuadro 3 se muestran las opciones de habilidades, conocimientos, competencias y formas de colaboración y trabajo ordenadas por valor de la estimación de mayor a menor. ${ }^{* * *},{ }^{* *}$, * indican el nivel de significación del $1 \%, 5 \%$ y $10 \%$, respectivamente. FUENTE: BBVA Research a partir de la OCDE.

correcta identificación de las habilidades más demandadas por los sectores líderes del crecimiento económico, con diagnósticos transparentes y actualizados a una demanda en constante evolución que permitan el continuo reciclaje y recapacitación de la fuerza laboral.

\section{Claves para afrontar la escasez relativa de habilidades}

Como señalaba Kaushik Basu, vicepresidente sénior y economista jefe del Banco Mundial en el prólogo de la publicación Doing Business 2015: Going Beyond Efficiency, existe el riesgo de que veamos el éxito de una economía solo a través de una visión macroeconómica centrada en las reformas fiscales, las medidas de índole monetaria y los programas de bienestar social. Sin embargo, resulta igualmente importante tener en cuenta otros factores que mantienen unido y afectan al engranaje que se encuentra por detrás del buen funcionamiento de una economía. Dentro de estos últimos elementos, podemos destacar aquellas instituciones y reglas que permiten a los 


\section{GRÁFICO 4}

DISTRIBUCIÓN POR OCUPACIONES, UE-28, ALEMANIA Y ESPAÑA, 2018

(En \% ordenadas de mayor a menor requerimiento de educación reglada)

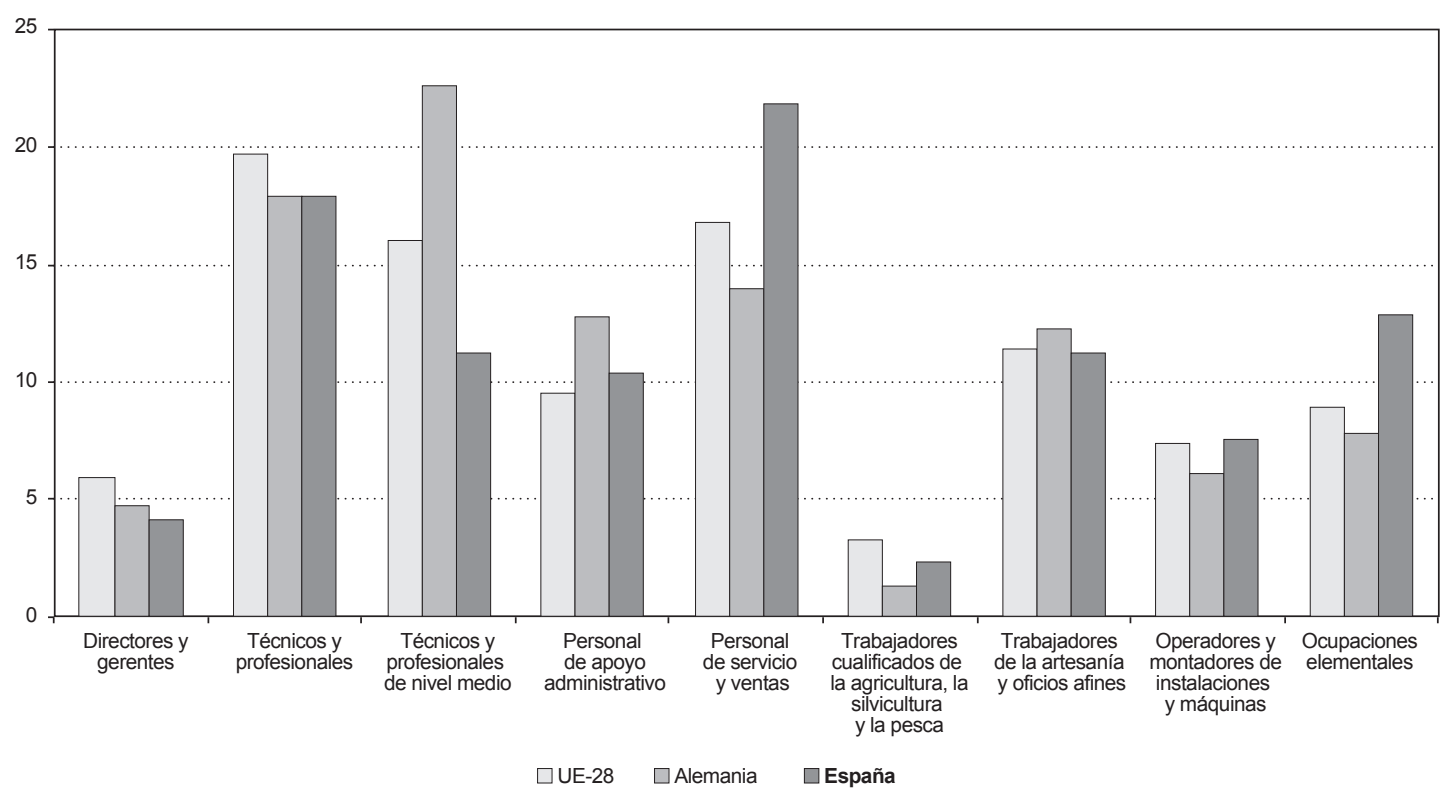

FUENTE: BBVA Research a partir de Eurostat.

agentes de una sociedad poder ajustarse y disponer de suficiente resiliencia ante los movimientos a escala mundial que están impulsando la disrupción digital y la globalización.

A modo de ejemplo, Heyman et al. (2019) realizan una retrospectiva de los factores que explican el buen comportamiento de la economía de Suecia desde hace un par de décadas. Entre los argumentos que han podido desarrollar las empresas se encuentran los incentivos y las recompensas para aumentar su capital humano, por ejemplo, con el aumento del nivel de formación de sus empleados y la mejora de los sistemas de retribución, así como con la contratación de personas más capacitadas y la adquisición de pequeñas empresas con alto nivel de capital humano y en expansión. Este tipo de actuaciones ha permitido mantener y expandir una elevada productividad, elemento básico en el crecimiento económico a largo plazo, así como ha favorecido que los factores productivos se hayan trasladado desde empresas de baja productividad a otras de mayor productividad, aumentando la eficiencia económica de la gestión de recursos.

Los resultados concuerdan con las necesidades que se han manifestado en el mercado laboral en España, y que viene derivado de una estructura educativa que ha fomentado la generación de una profunda dualidad formativa: una parte significativa de la distribución de la oferta de trabajo presenta un bajo nivel de estudios (Educación Secundaria Obligatoria) y otra parte dispone de un alto nivel de estudios (Educación Superior), mientras la distribución en el nivel medio es relativamente reducida. En cambio, la demanda por distintas cualificaciones muestra una distribución algo más uniforme. Como muestra el Gráfico 4 al comparar la distribución 
de puestos ocupacionales existente en el sistema productivo español, la estructura productiva en España no tiende a una alta polarización con puestos concentrados en ocupacionales vinculadas a una muy alta o muy baja cualificación².

Esta situación, que podemos calificar de corto plazo, está exigiendo que el desarrollo del conocimiento y los recursos educativos hagan un énfasis y un reforzamiento especial en la Formación Profesional y los estudios de nivel medio.

En este contexto de corto plazo no debe olvidarse la importancia de las transformaciones asociadas a la revolución tecnológica que ya está empezando a impactar en el propio tejido productivo de la economía española. Para atender no solo a las necesidades actuales sino también ir encauzando las futuras, que van a ir creciendo de forma inexorable, resulta necesario ampliar el estudio de la visión de las necesidades y las disponibilidades formativas más allá de las estrictamente técnicas.

Tanto los anteriores cambios tecnológicos como los nuevos que están por venir tienen al menos en común un factor: la formación. En consecuencia, cada vez resulta más necesario poner en el centro del desarrollo profesional de las personas la formación integral a lo largo de toda la vida del individuo. Si los cambios tecnológicos son generados cada vez en un período más reducido de tiempo, lo único que permanece en este proceso prácticamente continuo de disrupciones y cambios es el desarrollo de la persona a través de la formación.

Esta visión paradigmática de la formación no solo la debemos focalizar en los conocimientos, también resulta necesario en este momento donde tiene tanta importancia la comunicación y la información, ampliarla al desarrollo de las capacidades, competencias, habilidades

\footnotetext{
2 Dicho esto, se observa que en España se sobreponderan ocupaciones de baja cualificación (como el personal de servicio y venta, y las ocupaciones elementales) frente a ocupaciones de nivel medio (como técnicos y profesionales de nivel medio) cuando se comparan con Alemania.
}

y formas de colaboración y trabajo. En consecuencia, estamos priorizando sobre la base de las aptitudes y las actitudes de la persona (Diagrama 1).

El Estado necesita ser más consciente de la gran importancia que va a tener en la creación, el mantenimiento y el fomento de las grandes infraestructuras que subyacen a este proceso de formación integral.

Con el apoyo de las tecnologías digitales, es posible plantear un asesoramiento y unas guías personalizadas a la hora de realizar recorridos formativos. Las «autopistas formativas» vinculadas al conocimiento específico adquirido bajo la formación reglada pueden ser complementadas con "carreteras personalizadas» que permitan acceder a una mayor formación en aquellas aptitudes y actitudes transversales a toda área de conocimiento. En este contexto, resulta muy recomendable la implementación y desarrollo de hubs que permitan la conexión multidisciplinar de todas las aptitudes y actitudes del individuo.

Necesitamos que tanto las infraestructuras que lleven a los trabajadores a lo largo de este viaje como los vehículos empleados para llevarlo a cabo estén acondicionados. En la parte de infraestructuras y acondicionamientos subyacentes, se encuentran todos aquellos factores que influyen en el mercado de trabajo en España. En este mercado destacamos la necesidad de adaptación de las instituciones y normas en varias direcciones, siguiendo la línea de otros documentos, como Cardoso et al. (2016). Podemos destacar las siguientes propuestas que se centran en otros dos grandes agentes del mercado de trabajo: las empresas y la Administración pública.

Las nuevas tecnologías que están liderando el avance digital están generando una reorganización del trabajo en las empresas. Es recomendable aprender de la experiencia de aquellas empresas líderes que se están caracterizando por una innovadora gestión del talento y la adaptación de las estructuras empresariales a nuevas culturas, donde la inclusión y la diversidad son piezas claves en el engranaje de las mismas. También el impulso de las nuevas formas de trabajo y el uso de medios digitales 


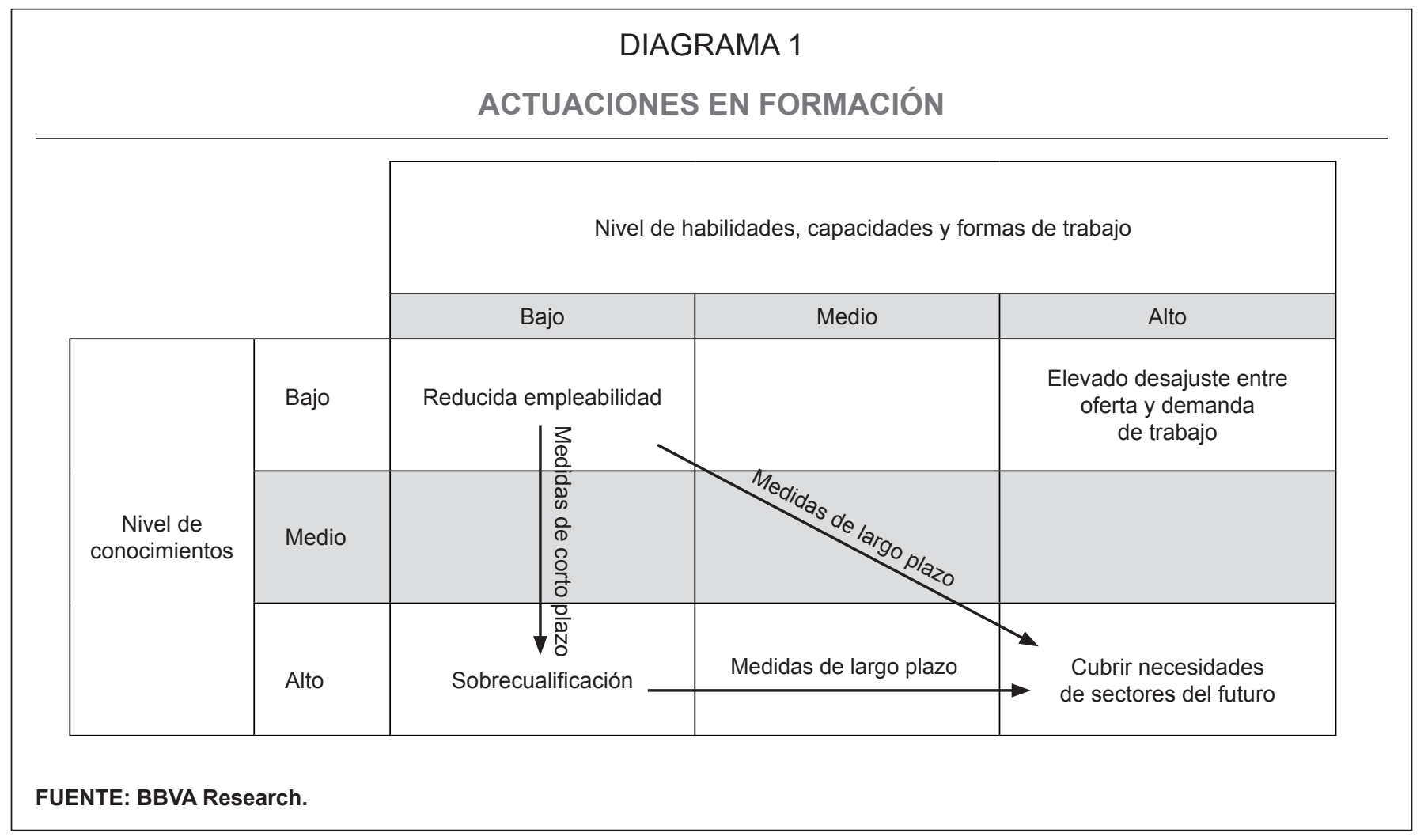

pueden ser una oportunidad para ayudar a reducir la dualidad contractual y la precariedad laboral.

El reto para la Administración pública es destacable. La tecnología y la globalización están cada vez más dificultando la implantación de soluciones que solo se circunscriben al marco nacional. Cada vez es más necesaria una respuesta global, a nivel de la Organización Internacional del Trabajo o de la Unión Europea. Sin embargo, todavía existe margen de actuación por el lado de la Administración pública, y podemos destacar la promoción de marcos regulatorios controlados al estilo de los denominados areneros o sandboxes que permitan realizar pruebas y generar experiencias que ayuden a establecer legislaciones adecuadas a la realidad laboral del siglo XXI, así como modernizar y hacer útiles las políticas activas de empleo en una economía en transición como la española. Por supuesto, la digitalización también puede ofrecer oportunidades para fomentar y facilitar el uso de estos instrumentos.
Desde el punto de vista de los acondicionamientos, resulta necesario promover medidas que ayuden a reducir la desigualdad económica y social, pues la escasez relativa de ciertas habilidades puede agravar el aumento de esta consecuencia tan desestabilizadora dentro de las sociedades actuales, como se ha comentado anteriormente en el caso de la informática. En el contexto laboral y dentro de un nuevo contrato social, la equiparación de derechos universales en materia laboral para toda la ciudadanía, con independencia del estatus jurídico del trabajador, puede constituir un instrumento potente que mitigue al menos estos efectos colaterales nocivos.

La Historia nos ha enseñado que las revoluciones tecnológicas mejoran el bienestar de las personas, pero solo tienen sentido cuando todos los integrantes de la sociedad se benefician de sus efectos. Es nuestra responsabilidad saber encontrar el modo de poder llevar a cabo este objetivo. 


\section{Conclusiones}

La transformación tecnológica que estamos viviendo parece exacerbar los desajustes laborales que llevamos experimentado en España durante las últimas décadas. En este contexto de incertidumbre, pero también de oportunidad, la economía española no ha sido capaz de reducir la elevada tasa de desempleo general (que esconde una gran heterogeneidad por grupos de trabajadores), la dualidad (formativa y contractual) laboral o el subempleo.

Con el objetivo de revertir esta tendencia y aprovechar las oportunidades que ofrece la era digital, proponemos dos grandes directrices de política, integrando en cada una de ellas una doble visión de corto y de largo plazo. Por un lado, utilizar la digitalización para profundizar en el análisis y seguimiento de las dinámicas del mercado laboral con el objeto de fortalecer mecanismos efectivos y de respuesta rápida que cuenten con una participación activa del sistema educativo. El objetivo sería permitir un desarrollo profesional a lo largo de toda la vida del individuo, basado en las actitudes y aptitudes de la persona. Por otro lado, el establecimiento de políticas centradas en aquellos colectivos específicos que puedan ser especialmente vulnerables durante la transición, para evitar que la transformación tecnológica sea vista como un juego de suma cero con perdedores y ganadores, en vez de una oportunidad de mejora del bienestar de todos los integrantes de la sociedad sin excepción.

\section{Referencias bibliográficas}

Andrés, J., Doménech, R., García, J. R., y Neut, R. A. (2019). El Impacto del Cambio Tecnológico y el Futuro del Empleo. BBVA Research. Mimeo.
Belapatiño, V., Iparraguirre, M., Ileri, A., Llanes, M. C., Mert, S. D., \& Posadas, C. (2019). Employment vulnerability to the digital revolution: some stylized facts on Argentina, Colombia, Mexico, Peru and Turkey. BBVA Research. Mimeo.

Bell, D. N. F., \& Blanchflower, D. G. (2018). Underemployment in the US and Europe. NBER Working Paper, (24927). Cambridge: NBER.

Cardoso, M., Doménech, R., García, J. R., Sicilia, J., y Ulloa, C. A. (2016). Hacia un mercado de trabajo más eficiente y equitativo. Observatorio BBVA Research. Observatorio Económico España, 6 de mayo de 2016. Recuperado de https://www.bbvaresearch.com/publicaciones/espana-hacia-un-mercado-de-trabajo-mas-eficiente-y-equitativo/

Díez Catalán, L., Doménech, R., y Neut, R. A. (2018). Informatización de sectores y rutinización de ocupaciones en España. Observatorio BBVA Research. Recuperado de https://www.bbvaresearch.com/publicaciones/ informatizacion-de-sectores-y-rutinizacion-de-ocupaciones-en-espana/

Doménech, R., García, J. R., Montañez, M., y Neut, R. A. (2018). Afectados por la revolución digital: el caso de España. Papeles de Economía Española, (156), 128-145.

Frey, C. B., \& Osborne, M. A. (2017). The future of employment: How susceptible are jobs to computerisation? Technological Forecasting and Social Change, 114, 254-280.

Heyman, F., Norbäck, P. J., \& Persson, L. (2019). The turnaround of the Swedish economy: lessons from large business sector reforms. CESifo Working Paper, (7627). Munich: Munich Society for the Promotion of Economic Research - CESifo GmbH. Available at https://www.ifo.de/ DocDL/cesifo1_wp7627.pdf

Mokyr, J., Vickers, C., \& Ziebarth, N. L. (2015). The History of Technological Anxiety and the Future of Economic Growth: Is This Time Different? Journal of Economic Perspectives, 29(3), 31-50.

Nordhaus, W. (2015). Are we approaching an economic singularity? Information technology and the future of economic growth. NBER Working Paper, (21547). Cambridge: NBER.

Turing, A. M. (1950). Computing Machinery and Intelligence. Mind, 49, 433-460. Available at https://www.csee.umbc. edu/courses/471/papers/turing.pdf 


\section{Sostenibilidad para la competitividad}

La sostenibilidad como factor de competitividad para la internacionalización de la pyme española

Medioambiente y desarrollo sostenible en Europa

La sostenibilidad en la política comercial como herramienta para la mejora de la competitividad

La incorporación de la agenda estratégica de los Objetivos de Desarrollo Sostenible como

Últimos números publicados:

Multinacionales en un cambiante contexto internacional

Economía de la Educación y Política Educativa

Números en preparación:

Internacionalización de las economías: nuevas formas, estrategias $y$ actividades factor determinante de la competitividad empresarial

Políticas de innovación desde la demanda y la consecución de los Objetivos de Desarrollo Sostenible: los retos del Gobierno como arquitecto de mercados y como comprador

Diversidad de género e innovación

Sostenibilidad y Objetivos de Desarrollo Sostenible: oportunidades para la competitividad y la colaboración en la industria de la moda española

La contribución de la sociedad civil a una economía sostenible

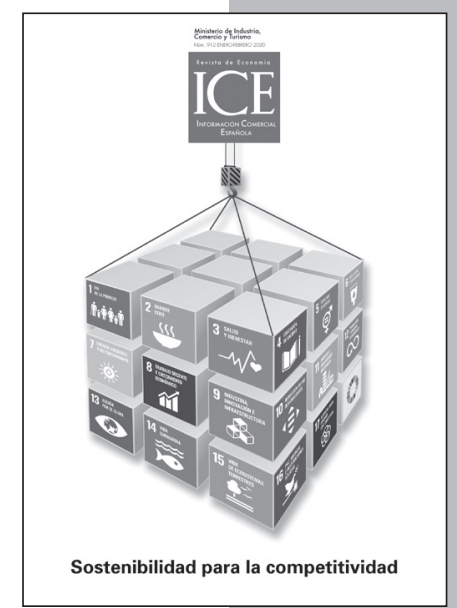

El uso de técnicas TIC innovadoras para la mejora de la Sostenibilidad.

Una valoración sobre las oportunidades abiertas 